\title{
Feature
}

\section{Video Views and Reviews: Cytokinesis: A Phenomenon Overlooked Too Often}

\section{Christopher Watters}

\author{
Department of Biology, Middlebury College, Bicentennial Way, Middlebury, VT 05753
}

Many students use "mitosis" and "cell division" interchangeably, apparently unaware that cell division includes mitosis as well as another, equally important process called cytokinesis. Mitosis separates duplicated chromosomes, whereas cytokinesis divides the parent cell (and duplicated chromosomes) into two daughter cells. Although these phenomena usually are linked temporally as well as spatially, mitosis can and does occur in the absence of cytokinesis (e.g., during the early development of Drosophila and other insects when many mitotic cycles occur before daughter nuclei become compartmentalized into separate cells; Alberts et al., 2002). Moreover, different mechanisms are responsible for each phenomenon. Thus, we give mitosis and cytokinesis different names, and it would be unfortunate (and especially confusing for students) if the definition of the former were expanded to include cytokinesis, as claimed in a recent review (Rieder and Khodjakov, 2003).

Thinking about these phenomena, I realized I was much more familiar with mitosis than I was with cytokinesis. Not only had I read contemporary reviews of the subject (Rieder and Khodjakov, 2003 [including 10 videos]; Mitchison and Salmon, 2001) and of mitosis Web sites (Blystone, 2003), I recently had reviewed videos and research articles dealing with the phenomenon in past Video Views and Reviews (Watters, 2003, 2002). My knowledge of cytokinesis, by contrast, was much less current and informed, and I was delighted when I had the opportunity in July 2004 to attend a conference on cytokinesis sponsored by the American Society for Cell Biology. This Feature arises from my experience at the conference and a subsequent search on High Wire Press (http://highwire.stanford.edu) for current research articles and videos on cytokinesis. Interested readers also may want to consult an older, comprehensive review written by a pioneer in the field (Rappaport, 1996) and the collaborative Web site maintained by the "Cytokinesis Mafia": http://www.bio.unc.edu/faculty/salmon/lab/ mafia/index.html.

As most students know, mitosis entails the condensation of duplicated chromosomes during prophase; their alignment and separation along the mitotic apparatus (or spindle, as it is sometimes called) during, respectively,

DOI: $10.1187 /$ cbe.04-08-0049

Address correspondence to: Christopher Watters (watters@ middlebury.edu). metaphase and anaphase; and the chromosomes' compartmentalization and relaxation during telophase. Once the chromosomes have been separated, cytokinesis begins, typically producing a cleavage furrow oriented at right angles to the axis of the spindle and passing through the plane of the metaphase plate. Anaphase, telophase, and furrow formation in a cultured animal cell are illustrated in Figure 1 (taken from Alsop and Zhang, 2003). Mechanistically, chromosomal movement occurs through the agency of the mitotic spindle, the kinetochores that attach chromosomes to various microtubular fibers of the spindle, the dynamic instability of microtubules themselves, and microtubular "motor" proteins (Rieder and Khodjakov, 2003). In contrast, cytokinesis and the formation of a cleavage furrow depend on the contraction of a cortical ring of actin and myosin filaments located just beneath the plasma membrane (Alberts et al., 2002). Ring constriction during cytokinesis is often referred to as purse-stringing, because the dividing daughter cells often resemble bags with gradually closing necks. The relative organization of the spindle and cortical ring is illustrated in Figure 2 (taken from Alsop and Zhang, 2004).

Although mitosis and cytokinesis are different phenomena, it is clear the mitotic apparatus is responsible for the location of the cortical ring midway between the spindle poles (centrosomes) and around the metaphase plate (Alberts et al., 2002). Micromanipulation of the spindle at critical times can cause the relocation of the cleavage furrow (see Rappaport, 1996). In the extreme, cytokinesis can occur in the absence of a spindle, as elegantly shown in an early experiment by Rappaport (1961), in any cytoplasmic region where astral microtubules from two centrosomes contact one another and adjacent regions of the plasma membrane. (This classic experiment is summarized in unattributed Figures 1831 [Alberts et al., 2002] and unattributed Figures 20-41 [Lodish et al., 2003].) It is not well understood how cortical ring contraction causes a furrow to form and to become constricted or how the spindle determines the location of the cortical ring.

Here I review recently published videos that depict the roles played by myosin II in contraction of the cortical ring during cellularization and cytokinesis in early development (Royou et al., 2004), by spindle and astral microtubules in regulating the formation of cleavage furrows during the cleavage of primary spermatocytes (Inoue et al., 2004), and 


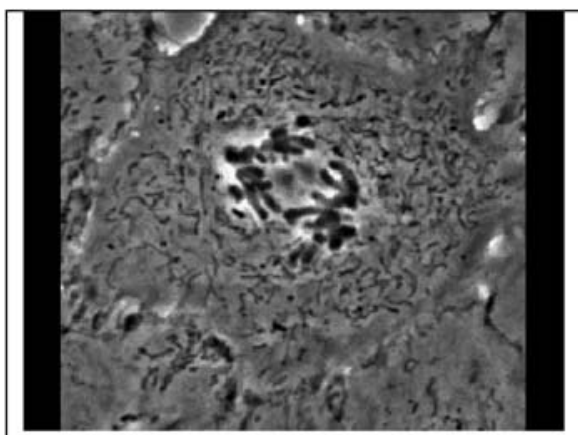

A

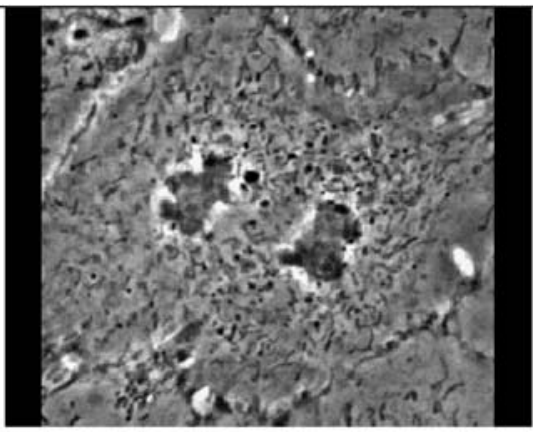

B

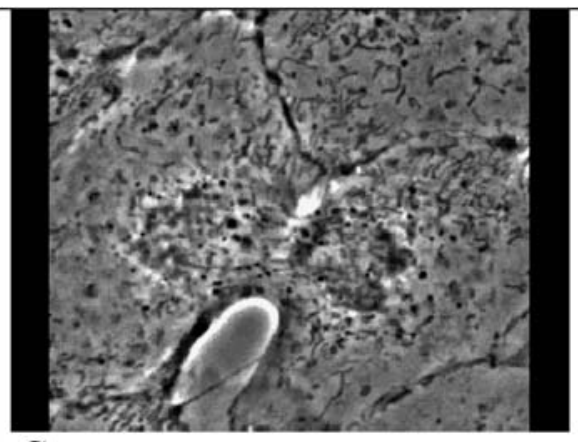

C

Figure 1. Phase contrast images taken from a time-lapse movie of a dividing cultured kangaroo rat kidney epithelial cell (PtK1) in late anaphase (A), telophase (B), and early interphase (C). Imagining a clock face superimposed on each image, the nearly invisible mitotic spindle is oriented along a line drawn from eleven to four o'clock and the cleavage furrow is forming at a right angle to the spindle along a line drawn from one to seven o'clock. Note the chromosomes being separated toward the spindle poles in A and becoming decondensed in B as nuclei reform. The furrow, especially evident in $\mathrm{C}$, is exaggerated in this material because in culture, the cells are flattened and not compacted in a three-dimensional tissue. The movie originated in the Salmon Laboratory and may be viewed on the Cytokinesis Mafia Web site: http:// www.bio.unc.edu/faculty/salmon/lab/mafia/phmit1.mov.

by a novel kinase in the regulation of cortical ring formation in postembryonic development (D'Avino et al., 2004). All three sets of videos were obtained from Drosophila material and nicely illustrate the complexity of cytokinesis in a single organism. For the sake of simplicity, however, I have focused on those aspects of the articles that relate to phenomena depicted in the videos. The videos and articles are suitable for intermediate and advanced undergraduate students as well as graduate students, and their study could be extended to cytokinesis in other organisms through a High Wire search.

I appreciate hearing your reactions to these reviews and your suggestions of other peer-reviewed videos for possible review as educational material.

\section{MYOSIN II, CELLULATION, AND CYTOKINESIS IN DROSOPHILA EMBRYOS}

Royou et al. (2004) examined the dynamic behavior of nonmuscle myosin II during cellularization and cytokinesis in early Drosophila embryos, using a chimera of green fluorescent protein (GFP) fused with the C-terminus of a myosin regulatory light chain (RLC). They also used rhodaminelabeled tubulin to localize microtubules (MT). Prior to studying myosin's role in cellularization, however, students might find it easier to appreciate myosin's role in forming cleavage furrows during cytokinesis (Figure 3), and the authors devote an experiment and the last of their five videos to myosin's contribution to cytokinesis. Most stu-

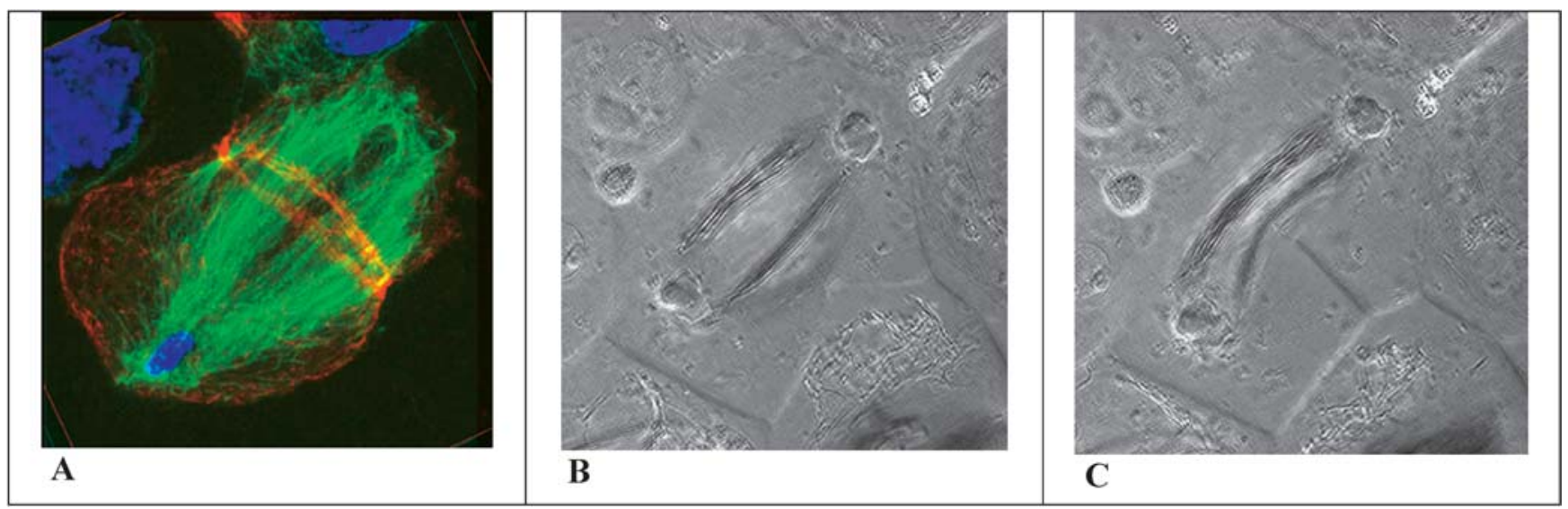

Figure 2. Terminal stages in the cell division of grasshopper spermatocytes illustrated in (A) a fluorescent image of a fixed preparation stained for DNA in the telophase nucleus (blue), MT in the mitotic apparatus (green), and actin primarily organized in a cortical ring (red); and phase-contrast images of a different spermatocyte with similar orientation, in telophase (B), and several minutes later as the cortical ring has contracted and the furrow has formed (C). The fluorescent image shows a 3-D composite reconstructed from 25 optical slices through the mitotic apparatus, which in the video slowly rotates about the $x$ - and $y$-axes: http://jcs.biologists.org/content/vol117/issue8/images/data/1591/ DC1/Movie3.mov. The time-lapse, phase contrast video may be viewed: http://jcs.biologists.org/content/vol117/issue8/images/data/1591/DC1/ Movie1.mov. Images reproduced with permission from The Company of Biologists and Journal of Cell Science. 


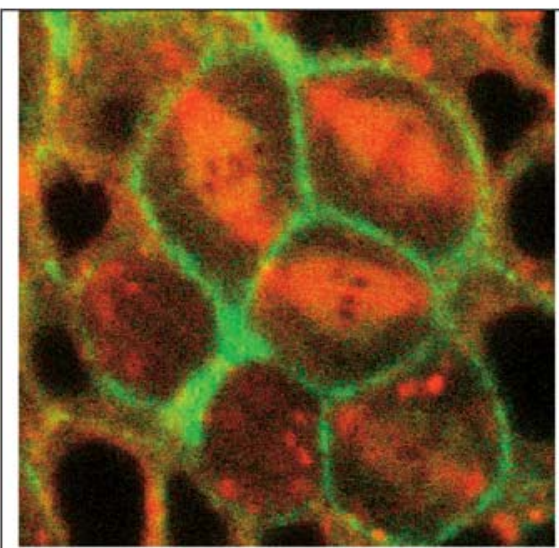

A

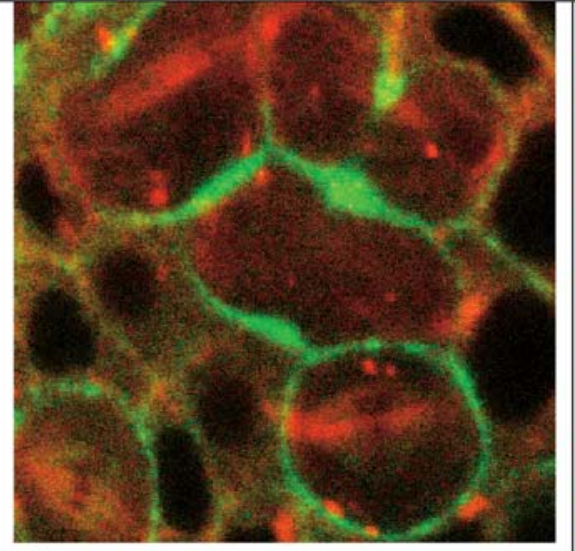

B

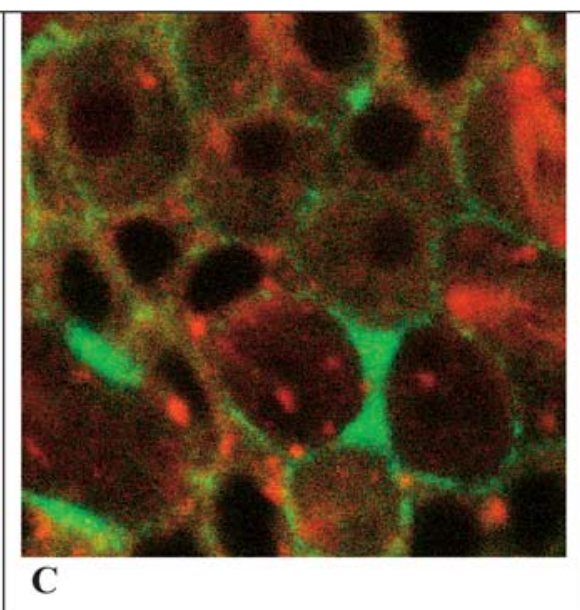

C

Figure 3. Confocal fluorescent images of cytokinesis in the cellular blastoderm of a Drosophila embryo viewed en face. Cells contain GFP-RLC (green) and rhodamine-labeled tubulin (red) illustrating the presence of myosin II and MT, respectively. Three cells in metaphase are imaged in (A), showing red mitotic spindles (and dark chromosomes) and green myosin diffusely located in thin, cortical regions just beneath the plasma membranes. As mitosis is completed (B and C), cytokinesis begins with myosin coalescing into cortical rings in the region formerly occupied by the metaphase plate. Each ring then begins constricting to form a cleavage furrow. In these very thin confocal images, the cortical rings appear in cross-section as two thickened nodules. The video may be viewed: http://www.molbiolcell.org/content/vol0/issue2003/images/data/ E03-06-0440/DC1/Movie5Fig8A.mov.

dents will recognize that actin (Figure 2) and myosin II colocalize in cortical rings and will have little difficulty hypothesizing a role for sliding filaments of actin and myosin during cortical ring constriction. Those students who are sticklers for detail might prefer to see the results of a colocalization experiment for actin and myosin in the same blastoderm cell, but the authors' data are consistent with the findings of others, as far as the composition of contractile rings in different cells is concerned (see Alberts et al., 2002). More intriguingly, Figure 3 (and authors' Movie 5) make it very clear that most of the myosin appears in the cortex just beneath the plasma membrane. Inquisitive students will want to know how this motor protein is targeted when it is first transported to the cortex following its synthesis on cytoplasmic ribosomes, and then during mitosis, when it is assembled further into a ring encircling the metaphase plate. They also might wonder whether actin polymerization and localization exhibits similar spatial and temporal patterns during ring formation. What moves the motors and the cytoskeleton and regulates their assembly? Data concerning the effects of an inhibitor of actin polymerization (cytochalasin) and of the spaghetti squash mutant (sqh $\left.{ }^{1}\right)$ lacking RLC provide useful information in this regard (authors' Figures $8 \mathrm{~B}$ and $\mathrm{C}$ ), but unfortunately, the source videos for these figures were not included in the archive. Authors' Movie 5 (and Figure 8A) nicely illustrates in control cells the temporal distinction between the formation of the contractile ring and its subsequent function, and it is likely students would find a comparison of this movie with videos of experimental cells very informative concerning the putative difference between formation of the cortical ring and its subsequent function. Finally, the very observant may notice small amounts of myosin apparently colocalizing with the mitotic spindle and wonder whether the motor protein also plays a role in mitosis.

The other four videos archived with Royou et al. (2004) concern the behavior of myosin II in cellularization. To understand this more complex phenomenon and the very striking videos, the viewer must first appreciate the idiosyncratic nature of early Drosophila development (Gilbert, 2003). Following fertilization, 13 rounds of mitotic division create a multinucleate (or syncytial) embryo in which nuclei first are evenly distributed throughout the very yolky, single-celled egg and then become localized around the periphery (Figure 4). At that time, cleavage furrows begin forming around the nuclei synchronously and perpendicular to the egg surface, to form the cellular blastoderm in a process called cellularization. Subsequent mitotic events during Drosophila development are accompanied by cytokinesis (as discussed in the previous paragraph).

At this point, I would encourage students to examine Movies 2 and 5 (and authors' Figures 2 and 8, respectively) and consider how cellularization compares with cytokinesis. Doing so provides an excellent test of their powers of observation and precision of thought. In Movie 2, once they recognize the dark, clear (fluorescence-free) nuclei and the egg surface highlighted by rhodamine staining, most students will appreciate that GFP-RLC (and presumably, myosin II) was recruited to the cortex as a preamble to cellularization, just as the chimera was during cytokinesis (compare Figures $3 \mathrm{~A}$ and $5 \mathrm{~A}$ ). And some may recognize contractile rings beginning to form, resembling tonsures at the apical surfaces of "bald" nuclei (Figure 5B). Most also will note large particles of GFP-RLC being transported from the egg's interior to these rings. With the recruitment of additional GFP-RLC, ring fluorescence intensifies and begins moving in a basal direction (toward the interior of the egg and the bottom of the video images; Figures 5C and D). Though invisible in the videos, cleavage furrows follow the ingression of these rings deep into the egg, and the authors report that after the rings and furrows advance beyond the nuclei, they begin to close at the basal side, partitioning nuclei into discrete cellular compartments. 


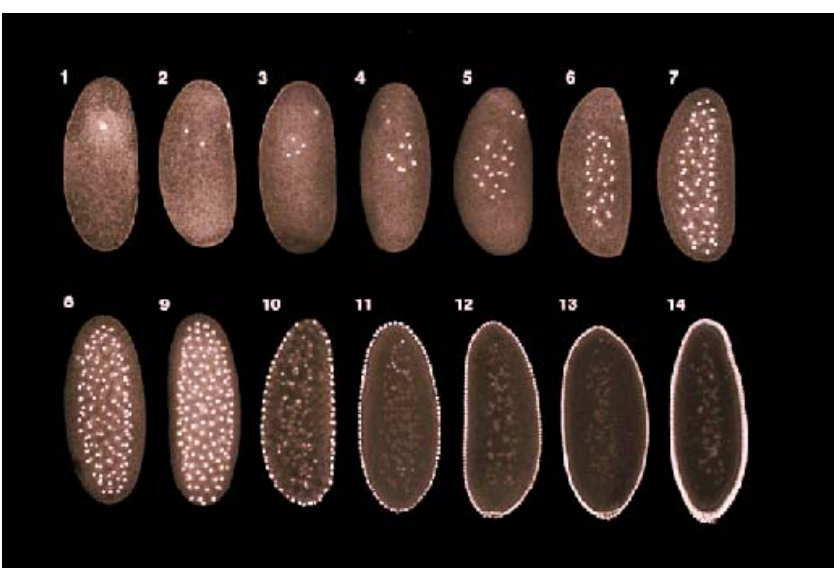

Figure 4. Confocal fluorescent images of labeled nuclei in an early Drosophila embryo during the first 13 rounds of mitosis. The first eight nuclear divisions occur centrally and in the absence of cytokinesis, after which the 256 nuclei migrate to the periphery of the egg and continue dividing. After 13 nuclear divisions, cleavage furrows begin to form around each nucleus, which creates a peripheral layer of cells surrounding a central core of undivided yolk. (Fig. 9.1, Developmental Biology, Seventh Edition, Gilbert, 2003: http://www.sinauer.com/milestones-devbio/Gilbert7e_263-303.pdf.)

The fluorescence, of course, is striking, and the hexagonal pattern of GFP-RLC localization during cytokinesis (Movie 5) resembles a similar image evident in Movie 3 toward the end of cellularization (when the cortical rings are imaged in a more en face manner than they are in Movie 2). Moreover, the rates of movement of green fluorescence evident in cytokinesis (Movie 5) and in the latter stages of cellularization in Movie 2 seem very similar, suggesting a similar mechanism for both. In this regard, more quantitative details about these rates would have been helpful, in the form of clock inserts showing elapsed time or descriptions of the time-lapse and projection rates. In fact, cellularization lasts approximately 50 minutes (according to the authors), whereas cytokinesis during later development takes only about 3 minutes (as inferred from authors' Figure 8A). The cells formed by cellularization are obviously much larger than those formed later in development by cytokinesis, but on the basis of the information available, one can only guess at the relative rates and any similarity of the underlying mechanisms. Moreover, cellularization appears to exhibit both slow and fast phases (Movie 2), with the fluorescent rings moving more slowly as they engirdle nuclei, and then more rapidly when closing.

At this point, most students may feel confused. On one hand, some of the very colorful aspects of cellularization and cytokinesis seem similar, especially the green honeycomblike patterns of cortical myosin localization common to both. Yet, the two phenomena differ remarkably in their global aspects. Not only does cellularization seem longer and biphasic, but it occurs in the absence of mitosis, unlike cytokinesis. During cellularization, there are no spindles or metaphase plates to orient the cortical rings. (As suggested by classical histology, the rings seem to form in association with centrosomes and astral MT fibers that are located at the apical surfaces of blastoderm nuclei [see Figure 9.3 in Gilbert, 2003]) Indeed, these rings engirdle intact nuclei as furrowing proceeds, and they contract (as during cytokinesis) only during the final stages of cellularization. (The last part of authors' Movie 3 presents an excellent view of pursestringing.) How does one make sense of these phenomena?

Heuristically, it may be worthwhile for students first to consider the possibility that cellularization and cytokinesis exhibit a similar sequence of steps or stages and then to hypothesize what these might be. One possible hypothesis contains the following sequence: 1) cortical recruitment of myosin and actin; 2) their assembly into a peripheral ring of fibers; 3) orientation or positioning of the ring; and, finally, 4) ring contraction. The two phenomena might then be understood to differ primarily in the length and prominence of the third step. During cytokinesis, cortical assembly and ring orientation around the metaphase plate seem to be rapid, nearly simultaneous events, whereas during cellularization, ring assembly occurs at the apical surface of a nucleus, and the ring then becomes oriented in a more protracted manner before contraction begins, by engirdling the nucleus and moving tens of microns in a basal direction in a process called ingression. Ring orientation and movement is also the prominent mechanistic feature of cellularization, because ingression of the cleavage furrow, which forms at the apical surface at the same time as the ring, seems tightly correlated with ring ingression. Following ingression, furrow closure during cellularization and both furrow formation and closure during cytokinesis require ring contraction, which is a relatively rapid event. Students are likely to raise many questions during their discussion. For example, are these four stages necessary and sufficient to describe both phenomena? Have some aspects of either phenomena been omitted? How might the existence of these stages be tested? Specifically, what causes and regulates step 3? The authors report the effects of cytochalasins and colchicine on cellularization, and examination of Movie 4 and authors' Figure 3 would prove instructive in answering this last question. So would the authors' data concerning the effect of the $s q h^{1}$ mutant on cellularization (authors' Figure 5).

As it turns out, ring movement during cellularization requires the presence of $\mathrm{MT}$, and although the ring must be present, actin and myosin do not seem to be actively involved in their own relocation. The MT requirement is consonant with older observations on fixed material showing the correlated elongation of blastoderm nuclei and the growth of MT and cleavage furrows (see Figure 9.3A in Gilbert, 2003). Students can conclude then, as do the authors, that furrow ingression and basal closure during cellularization are partially independent events. Reaching this conclusion then might lead to their wondering whether these two phenomena also might be partially independent, albeit concurrent, processes during cytokinesis.

Cellularization also provides a broad window for viewing other, more subtle aspects of cytokinesis, such as the increase in cell surface that accompanies cell division. More thoughtful students may wonder whether purse-stringing without the addition of new plasma membrane can separate daughter cells. In the case of cellularization, however, it should be obvious to most that furrow formation requires considerable expansion of the plasma membrane. The present study does not address how new membrane is added during cellularization, whether it is added during cytokinesis also, and in both instances, where the membrane might originate. 


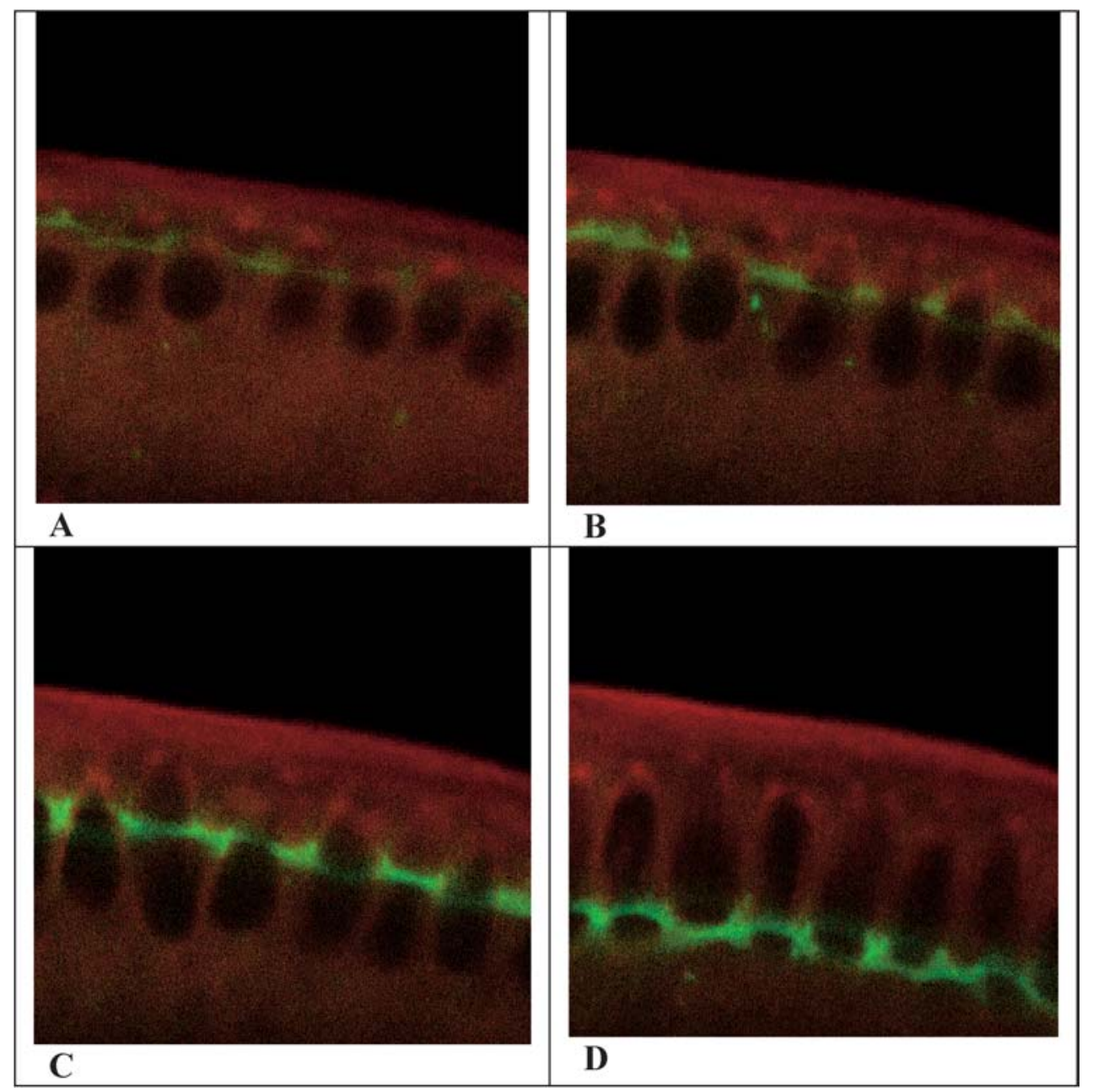

Figure 5. Confocal fluorescent images depicting cellularization, or the formation of the cellular blastoderm, in a section through the cortex of an early Drosophila embryo. Seven colorless, "black" nuclei near the cortex, which contains rhodamine-labeled MT (red) and GFP-RLC (green), are evident in A. The cortical rings begin to form in B and then begin to slide synchronously as "collars" past the nuclei in C and D. Cleavage furrows, which cannot be seen, form behind the advancing cortical rings. Note the nuclei become elongated as the cortical ring moves past them, suggesting they are being compressed as cellularization proceeds. The video may be viewed: http://www.molbiolcell.org/content/vol0/ issue2003/images/data/E03-06-0440/DC1/Movie2Fig2.mov.

Students querying the more general importance of MT in organizing cytokinesis will find the next two articles especially interesting.

\section{ROLE OF MICROTUBULES IN CYTOKINESIS}

Drosophila is an excellent system for the study of numerous developmental phenomena, in large part because of its detailed genetic history and the ease with which developmental mutants can be screened and propagated. Recently, Inoue et al. (2004) characterized the dynamics of spindle behavior and furrow formation in Drosophila primary spermatocytes that exhibited wild-type and orbit mutant phenotypes. They employed a chimeric protein consisting of GFP and $\beta$-tubulin to label the MT of the mitotic spindle, and they documented spindle and furrow behavior, using differential interference contrast (DIC) microscopy and fluorescence microscopy. As shown in other studies, the
Orbit protein localizes in various types of cell at the $(+)$ ends of MT and specifically at kinetochores, and orbit mutants often display disrupted spindles and incomplete mitoses arrested at metaphase (see Inoue $e t$ al., 2004). Selected images from videos of mitosis and furrow formation in spermatocytes from squash preparations are presented in Figure 6.

As is true in similar videos I have reviewed, simultaneously captured DIC and fluorescence images can be pedagogically impressive when the various optics have been optimized. The images of mitosis and cytokinesis in these very thin, squashed preparations are especially clear and distinct, and most students easily will recognize the mitotic spindle and the early stages of cleavage furrow formation in the paired control preparations of Video 1 (Figures 6A and B and Figures $6 \mathrm{C}$ and D, respectively). Two populations of MT are evident in the fluorescent images in late anaphase: a narrow cylinder of interior MT and the wing-like flaps of peripheral MT (Figure 6B). According to the authors, the 


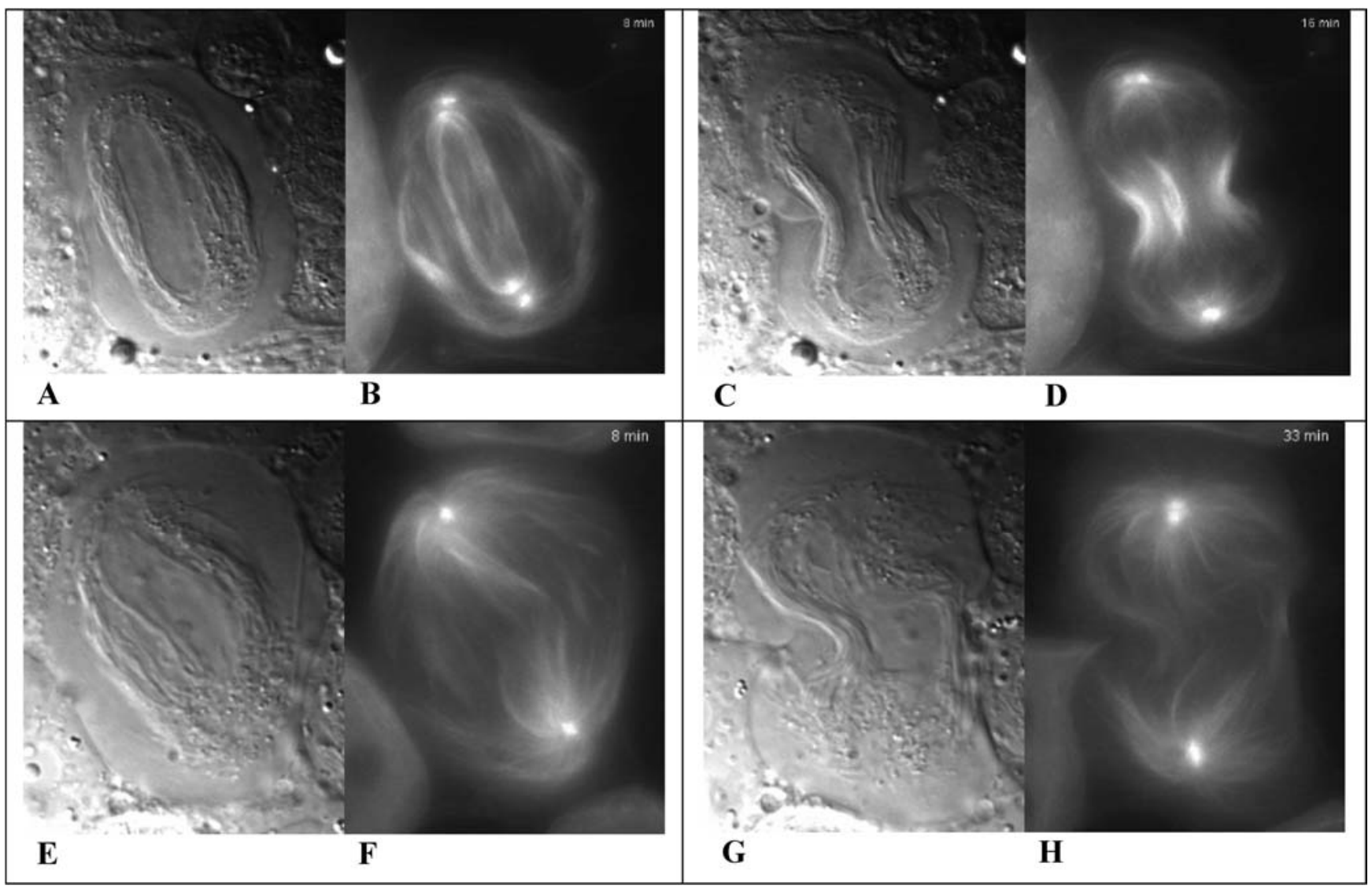

Figure 6. Images of primary spermatocytes during cytokinesis, from wild-type (A, B, C, D) and orbit mutant (E, F, G, H) Drosophila as viewed with DIC microscopy (A, C, E, G) and fluorescence microscopy (B, D, F, H), at various times after the onset of cytokinesis: at +8 min (A, B and E, F), $+15 \mathrm{~min}(\mathrm{C}, \mathrm{D})$ and $+33 \mathrm{~min}(\mathrm{G}, \mathrm{H})$. Comparable early $(8 \mathrm{~min})$ and late $(15$ and $33 \mathrm{~min})$ stages were selected from the videos. At an early post-anaphase stage, the mitotic spindle with its bright polar centrosomes is clearly evident in wild-type cells (B) but much less evident in the orbit mutant. Images A-D were taken from this video: http://www.jcb.org/cgi/content/full/jcb.200402052/DC1/4; images E-H were taken from this video: http://www.jcb.org/cgi/content/full/jcb.200402052/DC1/8.

latter are astral tubules that became segregated from the interior, spindle MT by remnants of the nuclear envelope, which in Drosophila apparently does not completely degrade during mitosis. As mitosis was completed, GFP fluorescence increased in the cell cortex, suggesting that the astral MT became bundled once they made contact with the cortex; most students will also note that fluorescence and bundling of the spindle MT increased in the midline as the furrow ingressed. Correspondingly, fluorescence of both interior and peripheral MT decreased at the spindle extremities, near the brightly fluorescent centrosomes, suggesting that both spindle and astral MT had detached from the centrosomal organization centers. Curious students may wonder whether other aspects of microtubular behavior were involved in these changes in fluorescence. Are the detached MT disassembling and/or being transported toward the spindle midline? How might alternative explanations be tested? Whatever the explanation, however, the pattern of microtubular behavior was drastically affected by the orbit mutant. (The mutants employed were hypomorphic, producing less than the wild-type amounts of Orbit protein, and students understanding this distinction may wonder whether the cell division phenotypes expressed in mutant cells might not be more variable than the observations reported.)

Several features of cell division in Drosophila spermatocytes changed in response to reduced levels of the Orbit protein, and characterizing these changes could form the basis of a student discussion of the mechanics of furrow formation and cytokinesis. The changes evident in Video 5 (Figures 6E-G) include an irregularly shaped spindle, apparently fewer spindle MT, less regular arrays of both interior and peripheral MT, and a failure of microtubular detachment at the centrosome poles as anaphase proceeded. In spite of these changes, a cleavage furrow formed, albeit more slowly, taking 18 minutes to reach a stage of ingression comparable to the wild-type cell (compare Figures 6G and $\mathrm{H}$ with Figures $6 \mathrm{C}$ and D). Purse-stringing in these mutants never advanced to the same degree as in the wild-type cells, however. Control cells formed highly fluorescent midbodies, which consisted of thin necks of cytoplasm densely packed with MT and connecting daughter cells (see the 25-minute frame of Movie 1 and authors' Figure 1). Moreover, according to the authors, the furrows in mutant cells later regressed. They argue that cleavage furrow formation (and cytokinesis) was initiated by astral 
(peripheral) MT, but completed through the intercession of spindle (interior) MT. The germinal importance of astral MT is further strengthened by events portrayed in Video 4, where a furrow formed parallel to the spindle (rather than perpendicular to it) and passed more or less along a plane through both the centrosomes! Having considered the importance of centrosomes during the early stages of cellularization (discussed previously) some students will find it interesting that astral MT may be involved in spermatocyte cell division. Some students will also be frustrated that neither movie of an orbit mutant cell (Videos 4 and 5) shows furrow regression, precluding their tracking the terminal failure of cytokinesis with other changes in microtubular behavior.

Responding to these movies and to the authors' suggestion that peripheral and spindle MT play different roles in cytokinesis, students likely will pose numerous questions concerning, for example, the location of Orbit in wild-type spermatocytes and the consequence of its absence in mutants. These latter concerns are addressed in authors' Figures 3, 6, and 7, respectively, using immunofluorescence of fixed preparations. In similar preparations of mutant cells, actin and Anillin (a contractile ring protein) failed to become localized in a ring surrounding the midregion of the spindle, and this result will confuse many students who associate the formation of a cleavage furrow with the presence of a contractile ring of actin, myosin, and such ancillary proteins as Anillin. How could the furrows seen in the video have been generated by incomplete rings? Confused students also might want to know how GFP chimeras of these dislocated proteins behave in squashes of living, mutant cells and how that behavior correlates with furrows that form and later regress in the mutants. More advanced students, aware of the kinds of GFPs available for tracking protein movement, would likely also be interested in tracking the behavior of a red fluorescent protein chimera of Orbit (YFP-Orbit) and GFP-tubulin coexpressed in wild-type cells. Inquisitive students also will wonder how the absence of a critical protein at the kinetochore or $(+)$ end of a spindle MT can affect the MT's attachment to the centrosome at its $(-)$ end. All in all, students will find this paper and movies very provocative.

Extending their examination of cytokinesis to spermatocytes of other insects, students may wish to consider the recent studies by Alsop and Zhang $(2003,2004)$ concerning the importance of spindle components in regulating the location of the contractile ring in grasshopper spermatocytes. These authors systematically analyzed the effects of asters, truncated spindles lacking chromosomes and asters, or MT alone. Cleavage furrows formed under all conditions, and their data suggest that when present in sufficient numbers (i.e., according to the authors, bundled!) MT alone are sufficient to reorient the cleavage furrow and stimulate its formation.

\section{KINASE REGULATION OF CYTOKINESIS: THE ROLE OF STICKY}

Given the rapidity with which the contractile ring forms and contracts during mitosis, and the apparent ease with which it can be relocated, it is not surprising this assemblage of actin, myosin II, and ancillary proteins is considered a very dynamic structure. In fact, students who have discussed the videos presented above likely would have queried the ring's dynamic features. If so, they will find the videos and article recently published by D'Avino et al. (2004) particularly interesting. These authors characterized the phenotype produced in cultured Drosophila S2 cells by RNAi silencing of sticky (sti), the gene coding for a protein kinase that regulates contractile ring behavior. The S2 line of cultured epithelial cells was derived from 20- to 24-hour embryos many years ago by Schneider (1969).

Images of a control cell (transfected with dsRNA for GFP) undergoing cytokinesis are presented in Figure 7, showing the beginning of furrow formation (Figure 7A), the completion of the furrow (Figure 7B), and much later, after interphase nuclei have been reformed in the daughter cells (Figure 7C), which continue to be linked by a midbody. When viewing the control video, students will find it useful to review the final steps of mitosis, the stages of cytokinesis, and how the two are related temporally.

While watching the video of cells transfected with sti dsRNA (Figures 7D-F), I would subdivide the students into two groups, asking one group to list the similarities of events portrayed in each video and the other group to characterize the differences. As the students compare their lists, they will refine their own language in order to convey what they have seen, which seems more pedagogically useful than telling them what they are seeing. This procedure also would reinforce what they have learned in the previous two sets of videos. And examining both the videos and the figures in the article could generate more discussion.

In Video 3, the most obvious effect of STI depletion is an apparent increase in the number of furrows generated, which produce cytoplasmic blebs of variable size (Figures $7 \mathrm{E}$ and $\mathrm{F}$ ), in addition to what appears to be a normal cleavage furrow formed at right angles to the mitotic spindle in the dividing experimental cell (Figure 7D). Most students who also examine Video 2, showing cytokinesis in another STI-depleted cell, might agree that although furrows formed in the inhibited cells, they were not as tightly regulated or spatially restricted in the experimental cells as in the control cell. To the extent that two daughter cells seemed to form, cytokinesis seemed temporally and spatially normal in both populations, at least based on what can be seen in the three archived videos (and authors' Figure 4 A). Unfortunately, students who also examine authors' Figure 3 and the related text discussion likely will become confused, because the authors conclude from their Figure 3 data that cytokinesis failed in the treated cells! Cytokinesis does indeed appear to have failed, because after 48 hours of RNAi treatment, $31 \%$ of the STI-depleted cells were binucleate, and after 72 hours of treatment, $60 \%$ of the cells were binucleate or multinucleate. Other students considering only the higher-magnification, fluorescent images in authors' Figures $4 \mathrm{~B}$ and $\mathrm{C}$ and in the videos would not be persuaded easily that cytokinesis failed, because the control and experimental cells depicted all seemed to have completed the process, and treated cells differed from controls mainly in the larger size of their midbodies and in the presence of blebs. Critical students are unlikely to be convinced by the authors' reasons for choosing to ignore the data in their Figure 4 and in the videos in favor of data presented in Figure 3. The apparent disagreement at this point could provide a foundation for a good discussion on 


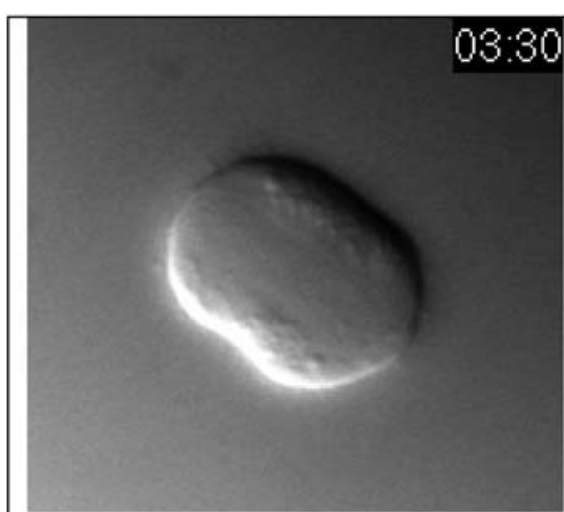

A

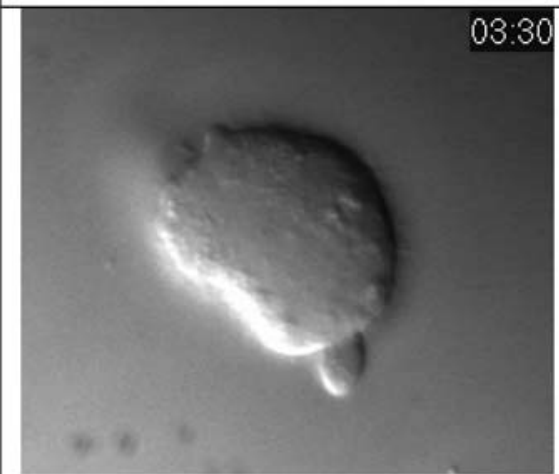

D

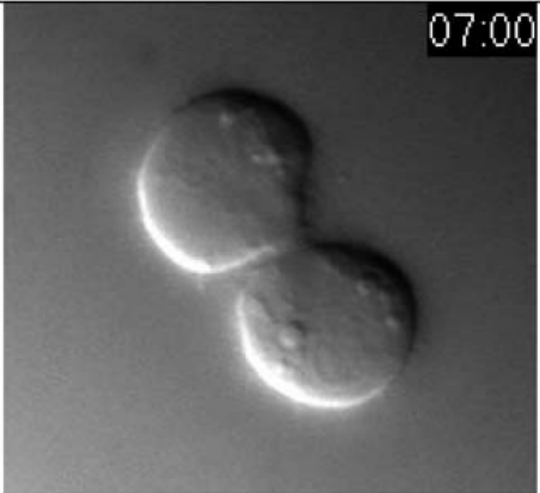

B

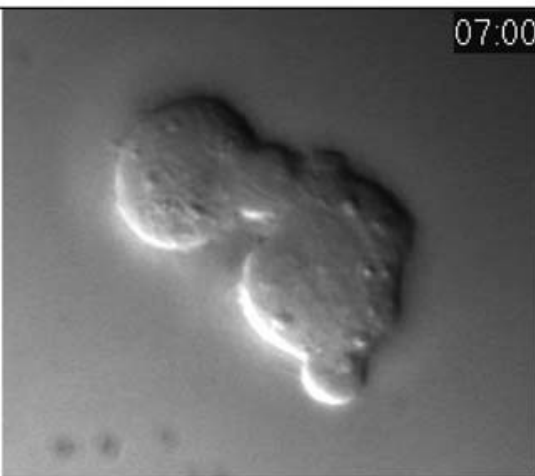

$\mathbf{E}$

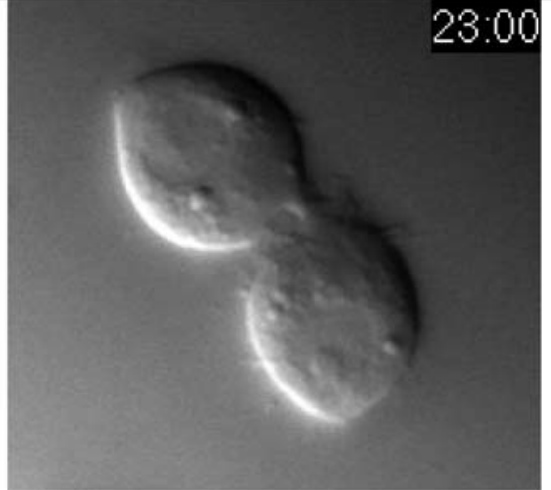

C

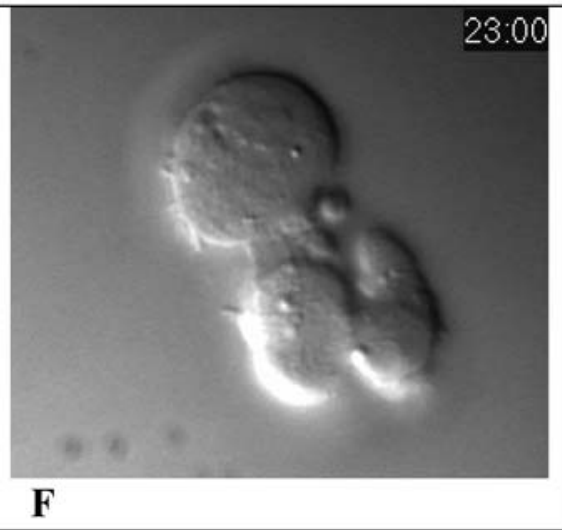

Figure 7. DIC images of cultured Drosophila S2 cells transfected with GFP dsRNA (control: A, B, C) or with sticky dsRNA (D, E, F) at the beginning of cytokinesis (A, D), the completion of cytokinesis (B, E) and after interphase nuclei (and nucleoli) have reformed in the control daughter cells $(\mathrm{C}, \mathrm{F})$. The clock inset indicates the real time elapsed since the onset of anaphase, in minutes and seconds. The control and experimental cells appeared identical at the onset of anaphase and their images at 00:00 are not presented. Images were collected at 15-s intervals and with a QT playback rate of 6 frames per second, the events portrayed are speeded up 90 -fold. The video of the control cell may be viewed: http://www.jcb.org/cgi/content/full/jcb.200402157/DC1/2; and for the experimental cell: http://www.jcb.org/cgi/content/full/ jcb.200402157/DC1/4.

the reproduction of data, sampling errors in small data sets and the variability of RNAi treatment among different experiments.

In any event, most observers would likely agree the cortical rings appear to have contracted normally in both control and STI-depleted cells, and it would be useful to postulate how cytokinesis could have been successful in the former instance, but could have failed in the latter. To formulate hypotheses, students likely will want to know where STI is located normally and whether its expression is restricted and/or its location affected in RNAi-treated cells. Authors' Figure 5 provides useful data in this regard, but students also may wonder about the variability of STI in RNAi-treated cells. Some students also may want to know what happens to midbodies at the end of cytokinesis and whether midbody scission occurs in control cells, but presumably fails in treated cells. This is a crucial point, because one resolution of the paradox raised by the apparently contradictory data in authors' Figures 3 and 4 (and the videos) entails the dissolution of contractile rings in both populations. Such disassembly would be inconsequential (and normal) in control cells already completely separated by scission, but the constricted midbodies of incompletely separated, treated cells could secondarily expand following ring disassembly, resulting in the formation of large, binucleate cells as seen in Figure 3. Unfortunately, midbody scission is not addressed in this paper and little seems to be known about the process. Perceptive students who have read the article closely, however, may also note that Anillin, an actin-binding protein thought to link the contractile ring to the plasma membrane (Somma et al., 2002), is localized in the scission zone of control cell midbodies, but more broadly dispersed in sti RNAi-treated cells. Such dislocation could account for the failure of membrane fusion that must accompany the final stages of scission, and it would be interesting to learn whether STI and Anillin interact, and, given the kinase function of STI orthologs in other cells, whether Anillin is phosphorylated in control cells and dephosphorylated in sti RNAi-treated cells. Finally, based on genetic data (authors' Figure $6 \mathrm{H}$ ), the authors infer that one of the kinase targets of STI was the myosin RLC coded by the spaghetti squash gene. Thoughtful consideration of this effect could produce an alternative hypothesis: Phosphorylated RLC produces a more stable contractile ring than the dephosphorylated form thought to result from sti iRNA-treatment. If true, multi- 
nucleation of treated cells could have resulted from premature contractile ring disassembly and not from the absence of scission.

Testing these alternative hypotheses - indeed, understanding how contraction of an actomyosin ring actually separates one cell into two-requires more visual and molecular information about the final steps of cytokinesis than has been provided by any of these videos or papers. Final discussion of these papers, therefore, might well involve hypothesisbuilding and literature searches concerning other instances of membrane expansion and fusion.

\section{REFERENCES}

Alberts, B., Johnson, A., Lewis, J., Raff, M., Roberts, K., and Walter, P. (2002). Molecular Biology of the Cell, 4th ed., New York: Garland Science.

Alsop, G.B., and Zhang, D. (2003). Microtubules continuously dictate distribution of actin filaments and positioning of cell cleavage in grasshopper spermatocytes. J. Cell Sci. 117, 1591-1602.

Alsop, G.B., and Zhang, D. (2004). Microtubules are the only structural constituent of the spindle apparatus required for induction of cell cleavage. J. Cell Biol. 162, 383-390.

Blystone, R. (2003). WWW.Cell Biology Education. Cell Biol. Ed. 2, 214-219.

D'Avino, P.P., Savoian, M.S., and Glover, D.M. (2004). Mutations in sticky lead to defective organization of the contractile ring during cytokinesis and are enhanced by Rho and suppressed by Rac. J. Cell Biol. 166, 61-71.

Gilbert, S. (2003). Developmental Biology, 7th ed., Sunderland, MA: Sinauer Associates.
Inoue, Y.H., Savoian, M.S., Suzuki, T., Mathe, E., Yamamoto, M.-T., and Glover, D.M. (2004). Mutations in orbit/mast reveal that the central spindle is comprised of two microtubule populations, those that initiate cleavage and those that propagate furrow ingression. J. Cell Biol. 166, 49-60.

Lodish, H., Berk, A., Matsudaira, P., Kaiser, C.A., Krieger, M., Scott, M.P., Zipursky, S.L., and Darnell, J. (2003). Molecular Cell Biology, 5th ed., New York: W.H. Freeman.

Mitchison, T.J., and Salmon, E.D. (2001). Mitosis: a history of division. Nat. Cell Biol. 3(1), E17-21.

Rappaport, R. (1961). Experiments concerning the cleavage stimulus in sand dollar eggs. J. Exp. Zool. 148, 81-89.

Rappaport, R. (1996). Cytokinesis in Animal Cells. Developmental and Cell Biology Series, ed. P.W. Barlow, J.B.L. Bard, P.B. Green, and D.L. Kirk. Cambridge, UK: Cambridge University Press.

Rieder, C.L., and Khodjakov, A. (2003). Mitosis through the microscope: advances in seeing inside live dividing cells. Science 300, 91-96.

Royou, A., Field, C., Sisson, J.C., Sullivan, W., and Karess, R. (2004). Reassessing the role and dynamics of nonmuscle myosin II during furrow formation in early Drosophila embryos. Mol. Biol. Cell. 15, 838-850.

Schneider, I. (1969). Cell lines derived from late embryonic stages of Drosophila melanogaster. J. Embryol. Exp. Morphol. 27, 353-365.

Somma, M.P., Fasulo, B., Cenci, G., Cundari, E., and Gatti, M. (2002). Molecular dissection of cytokinesis by RNA interference in Drosophila cultured cells. Mol. Biol. Cell. 13, 2448-2460.

Watters, C. (2002). Video Views and Reviews. Cell Biol. Ed. 1(2), 111114.

Watters, C. (2003). Video Views and Reviews. Cell Biol. Ed. 2(1), 913. 